\title{
Another year of achievement for OEM
}

\author{
Dana Loomis, ${ }^{1}$ Malcolm Sim ${ }^{2}$
}

Occupational and Environmental Medicine passed several notable milestones in 2009, beginning with the appointment of a new editorial team after the previous editor in chief, Keith Palmer, stepped down at the end of 2008.

In July, we announced ${ }^{1}$ that the entire back archive of OEM and the British Journal of Industrial Medicine, right back to volume 1, number 1 in 1944, was freely available to the research community through our web site (http://oem.bmj.com/content/by/year). The extraordinary value of this resource was emphasised in three insightful editorials in the same issue highlighting groundbreaking papers published during the journal's long history. ${ }^{2-4}$

We announced another important development in October, when we reported that OEM's impact factor had risen to 3.3 in $2008 .^{5}$ With this increase, $O E M$ has maintained its number one ranking among all journals focussing on occupational health and moved up to 16 th in the larger category of over 100 journals covering public, environmental and occupational health. We thank our authors and our volunteer reviewers for their contributions to the journal's rise.

The journal's increasing prominence is good news for our authors and readers, but

\footnotetext{
${ }^{1}$ School of Public Health, University of Nevada, Reno, Nevada, USA ${ }^{2}$ Department of Epidemiology and Preventive Medicine, Centre for Occupational and Environmental Health, Monash University, Melbourne, Australia

Correspondence to Dana Loomis, School of Public Health - 274, University of Nevada, Reno, Nevada 89557-0274, USA; dploomis@unr.edu
}

it has also made publishing in $O E M$ more competitive. We received over 720 papers in $2009-a 30 \%$ increase relative to the previous year. The number of pages we can print in a year has not increased, however, so this inevitably means a decrease in the proportion of papers we can accept. We estimate that, when the final figures for 2009 are available, we will have accepted about $20 \%$ of the papers submitted, compared to $27 \%$ in 2008 and $33 \%$ in 2007.

More submissions mean more work for the editors, but we are still continuing to increase our service to the worldwide occupational and environmental medicine community. For authors, we have progressively shortened the time it takes to reach decisions on submitted papers. Every new contribution to OEM is reviewed by the editor in chief or the deputy editor (and often by both of us), and authors will normally know within a week whether we have decided to send a paper on for review or reject it. In 2009 we also introduced enhanced Online First publication. Original articles, short reports, reviews, commentaries and correspondence are now all published electronically before they appear in print. In addition, the online versions are now formatted and typeset before being posted. A further benefit is that the online publication usually occurs within about 3 weeks of acceptance.

For the research and practice communities and other readers, we plan to continue to provide authoritative editorials discus- sing developments in the field and commentaries on papers in the journal, in addition to publishing high quality original research and reviews. We have also launched a new effort to strengthen the evidence base for occupational medicine practice. $^{6}$ This will include inviting more original research articles and reviews that can help occupational health care providers make better decisions. In 2010, we also plan to add a "Cochrane Corner" section, which will highlight new systematic reviews and other developments from the Cochrane occupational health field.

We are pleased with these new developments and think they will further increase the journal's impact. We also want to hear directly from our readers about the kind of content and services you value most in the journal. You can comment by sending a rapid response to this editorial through our web site.

\section{Competing interests None.}

Provenance and peer review Commissioned; not externally peer reviewed.

Accepted 17 December 2009

Occup Environ Med 2010;67:73.

doi:10.1136/oem.2009.054551

\section{REFERENCES}

1. Loomis D. Sixty-five years of the Journal go online. Occup Environ Med 2009;66:425-6.

2. Newman Taylor A. Asbestos, lung cance and mesothelioma in the British Journal of Industrial Medicine. Occup Environ Med 2009;66:426-7.

3. Lynge E. From cross-sectional survey to cohort study Occup Environ Med 2009;66:428-9.

4. Rappaport SM. Assessing workplace exposures: turning to the past for guidance. Occup Environ Med 2009;66:429-30

5. Loomis D. OEM's impact factor continues moving upward. Occup Environ Med 2009;66:641.

6. Sim MR, Agius R. The role of occupational and environmental medicine in strengthening the evidence base for occupational health practice. Occup Environ Med 2009;66:570-1. 